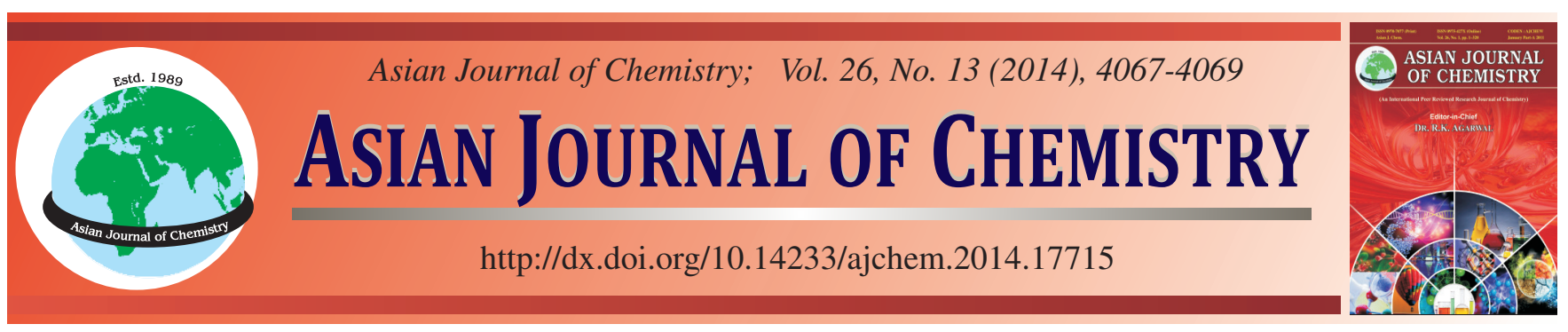

\title{
On-Line and Real-Time Analysis of Moisture Content in Activated Carbon Powder by X-Ray Scattering Technique $\nmid$
}

\author{
Yong Suk Chor ${ }^{1}$, JaEsik Hwang ${ }^{2}$ and Jong-Yun KIM ${ }^{1, *}$
}

${ }^{1}$ Nuclear Chemistry Research Division, Korea Atomic Energy Research Institute, 989-111 Daedeok-daero, Yuseong-gu, Daejeon, 305-353, Republic of Korea

${ }^{2}$ Korea Institute of Industrial Technology, 89, Yangdaegiro-gil, Ipjang-myeon, Seobuk-gu, Cheonan-si, Chungcheongnam-do, 331-822, Republic of Korea

*Corresponding author: Fax: +82 42 8688148; E-mail: kjy@kaeri.re.kr

On-line and real-time analyses of moisture in activated carbon powders were performed using a pilot-scale X-ray monitoring system. The system was composed of an X-ray tube generator as an X-ray source, a cadmium telluride (CdTe) X-ray detector and a circular rotating disk to simulate real processes. Total X-ray scattering intensities from the powder samples moving at a speed of $4 \mathrm{~m} / \mathrm{min}$ were measured with a very short counting time of $0.1 \mathrm{~s}$. The wet powder samples with various moisture contents were successfully monitored in real-time using our in-house built X-ray scattering measurement system.

Keywords: Real-time, On-line, X-Ray, Moisture, Activated carbon.

\section{INTRODUCTION}

Process analytical chemistry is an emerging field of modern analytical chemistry and process engineering for the quality control of the intermediate and final products ${ }^{1-3}$. In situ real-time analysis in the process analytical chemistry is essential for process control capable of early fault detection $^{1}$. We developed an X-ray scattering technique to determine the moisture content of the silica powders and the thickness of organic films ${ }^{4,5}$. In the present study, a realtime analysis of activated carbon powders was performed using an in-house built pilot-scale X-ray monitoring system for the determination of moisture content in the powder. Activated carbon is an attractive material in many industries such as waste water treatment, pollutant purification, solvent recovery, catalysis, etc. ${ }^{6-9}$. The effect of moisture content in activated carbon on the performance as an adsorbent should be carefully considered because there have been plenty of reports with regard to the synergistic or adverse effect of moisture with respect to the specific process characteristics. Thus, the process monitoring of the moisture in the activated carbon is very valuable for the precise control of the moisture content in the powder processes.

\section{EXPERIMENTAL}

An X-ray measurement system for the determination of moisture content in the activated carbon powders is depicted in Fig. 1. The in-house built measurement system was composed of an X-ray tube generator as an X-ray source, a cadmium telluride (CdTe) X-ray detector connected to a multi-channel analyzer (X-123-CdTe, AMPTEK, USA) and a circular rotating disk with a trench of $10 \mathrm{~cm}$-in depth and $20 \mathrm{~cm}$-wide. The powder samples were placed in the trench of the rotating disk operated at a speed of $1 \mathrm{rpm}$ to $60 \mathrm{rpm}$ (linear velocity 4 $\mathrm{m} / \mathrm{min}$ for $1 \mathrm{rpm}$ ). The energy calibration of the 1024-channel CdTe detector was not performed since the exact information on the peak energy of the X-ray spectra is not important in this study. Instead, the total sum of the count rates by the scattered radiation over the whole range of the channels was used to identify the variation of the moisture content qualitatively.

Activated carbon powder (SGF100, Samchully Pharm. Co., Korea) was used as a model powder for the determination of the moisture content using an X-ray. For the preparation of the activated carbon powder containing a certain amount of moisture, a plastic bottle containing the predetermined amounts of solid powder and water was heated for $1 \mathrm{~h}$ at $100^{\circ} \mathrm{C}$ and 


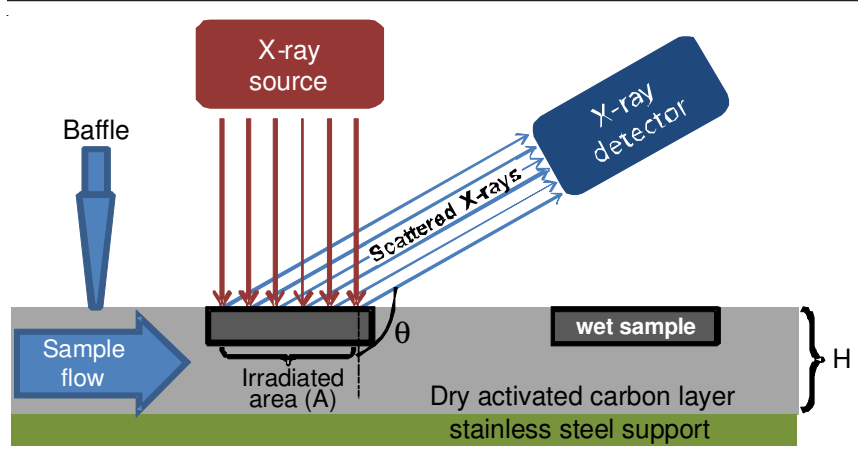

Fig. 1. On-line X-ray measurement system for the determination of moisture content, where $\mathrm{H}$ is the thickness of the powder samples, $\mathrm{A}$ is the irradiated area and $\theta$ is scattering angle

then mixed using a roller for the complete mixing. Eight activated carbon sample trays with 15, 20, 25, 30, 35, 40, 45 and $50 \mathrm{wt} \%$ water were placed as shown in Fig. 1.

For optimization of the X-ray measurement system, the effects of the irradiated area, the scattering angle and the tube voltage were investigated to find the optimum conditions of the X-ray measurement system. For more reproducible results, the distance between the X-ray source and the powder surface was controlled at a constant level using a baffle.

\section{RESULTS AND DISCUSSION}

Parameters affecting the X-ray scattering intensity: If the samples are irradiated with X-rays, an X-ray undergoes several interactions with the atomic electrons such as photoelectric absorption, coherent Rayleigh scattering, incoherent Compton scattering and Thomson scattering. X-Ray also exhibits typical wave properties such as interference, diffraction, reflection, refraction and polarization. In general, the scattered X-ray intensity is affected by many factors such as scattering angle $(\theta)$, irradiated area, tube voltage and current.

Fig. 2 shows the effect of scattering angle on the total count rate of all scattered $\mathrm{X}$-ray radiation collected by the X-ray detector. The scattered X-ray intensity increased as the scattering angle increased from 133 to $155^{\circ}$. As the scattering angle increases, the Rayleigh scattering intensity decreases while the Compton scattering intensity increases. Because the intensities of both Rayleigh-scattered radiation (a coherent scattering by the atomic field) and Compton-scattered radiation (incoherent scattering with an atomic electron) increases linearly with the moisture content ${ }^{4}$, the scattering count rates were integrated over the entire range of channels. The total sum of the scattering intensity increased from $82 \pm 4-105 \pm 6 \mathrm{kcps}$ as the scattering angle increased from 133 to $155^{\circ}$ in Fig. 2 . The tube voltage exhibited the same feature as for the scattering angle as shown in Fig. 3. It is clear that the higher scattering angle and the higher tube voltage are better for the larger intensity measurement as expected from the previous studies on the angular distribution of the scattering intensities of $\mathrm{X}$ rays and the effect of the tube voltage on the X-ray intensity ${ }^{10,11}$.

The total sum of the scattered X-ray intensities was linearly dependent on the area of the X-ray irradiation as shown in Fig. 4, although the detector area is only $9 \mathrm{~mm}^{2}$, which is very small compared to the radiated area. The irradiation area should be carefully controlled with respect to the purpose of

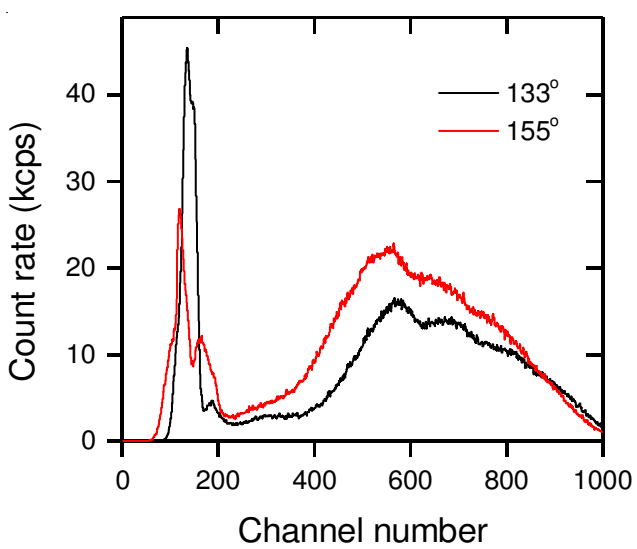

Fig. 2. X-Ray spectra of the activated carbon powder measured using $\mathrm{CdTe}$ detector and the effect of scattering angle $\left(\theta=133\right.$ and $\left.155^{\circ}\right)$ on the scattered intensity

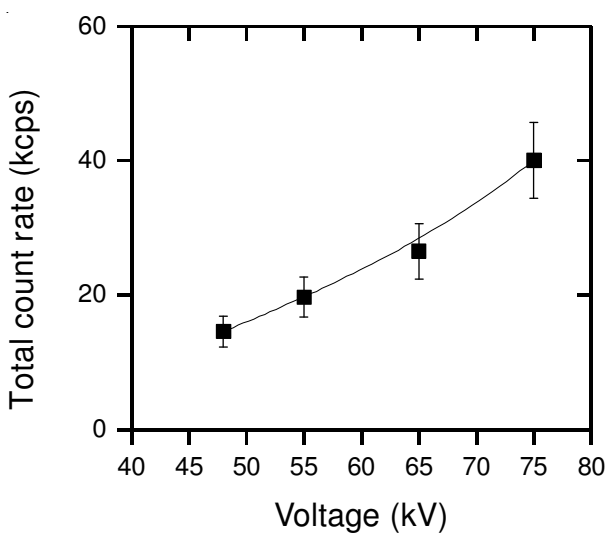

Fig. 3. Effect of tube voltage on the scattered X-ray intensity

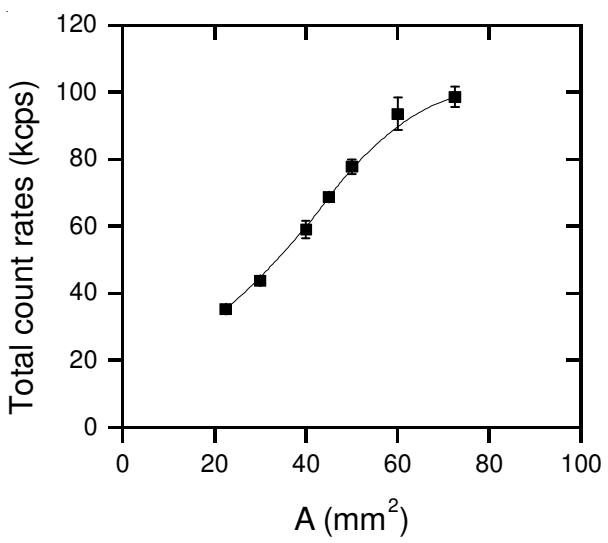

Fig. 4. Effect of the irradiated area on the scattered X-ray intensity

each field process monitoring. If the area is too large, it is less probable to detect the local variation of the process samples, while if it is too small, it is more probable to increase the falsealarm rates. Multiple detectors will be one possible solution to avoid such a false-alarm.

On-line and real-time monitoring of moisture content: In a majority of field processes, the key to the successful operation of the process is the identification of the fault detection as early as possible. The limit of detection and the sensitivity of the X-ray scattering technique are dependent on the performance of the X-ray measurement system and the structural properties of the powder such as the pore size and surface area. In addition, the counting time becomes critical if the 
sample is moving at a high speed. Fig. 5 shows that the eight wet samples with $15-50 \mathrm{wt} \%$ water could be identified clearly by the eight distinct peaks in the time $v s$. count rate curves, at the condition that the wet sample tray was moving at a speed of $4 \mathrm{~m} / \mathrm{min}$, the distance between samples was $0.5 \mathrm{~m}$ and the counting time was $0.1 \mathrm{~s}$. Each peak intensity corresponding to the scattered radiation by the $50-15 \mathrm{wt} \%$ water in the activated carbon decreased as the moisture content decreased. However, the quantitative correlations were not investigated in the present study.

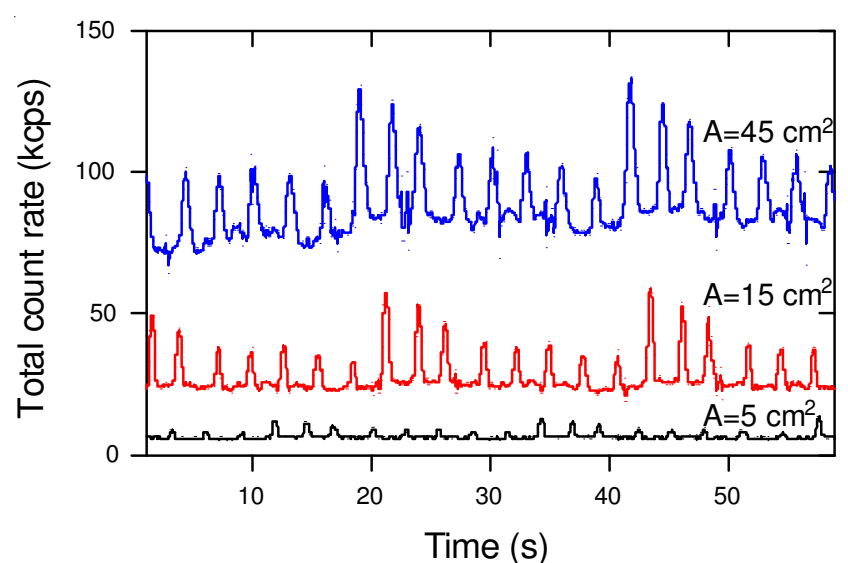

Fig. 5. Variation in the total X-ray scattering intensities with the time of flow $(\mathrm{t})$ for the wet activated carbon samples moving at a speed of $4 \mathrm{~m} / \mathrm{min}$

\section{Conclusion}

To date, on-line methods such as infra-red (IR) spectroscopy and off-line methods such as Karl-fisher titration have been used as a monitoring technique in the field process. Nevertheless, those conventional methods are not appropriate as a real-time monitoring technique in some harsh conditions of the field processes or a process with a fast-moving conveyor belt, which is one of the most convenient and efficient tools for the transportation of powder materials from one place to another. In particular, IR spectroscopy cannot be applied to the black-coloured carbonaceous materials. In principle, our current X-ray scattering technique using a very short counting time can be applied to any type of material in on-line and realtime monitoring of the moisture content in a powder sample. The quantitative monitoring is beyond the scope of this study and a further study might not be necessary in cases that the "calibration-free" monitoring is used for a qualitative fault detection of the field processes.

\section{ACKNOWLEDGEMENTS}

This work was supported by the Nuclear Research and Development Program through National Research Foundation of Korea (No. 2014000288) funded by the Ministry of Science, ICT and Future Planning.

\section{REFERENCES}

1. J. Workman Jr., B. Lavine, R. Chrisman and M. Koch, Anal. Chem., 83, 4557 (2011).

2. T.Y. Zhang, Chem. Rev., 106, 2583 (2006).

3. R.M. Balabin and S.V. Smirnov, Analyst, 137, 1604 (2012).

4. Y.S. Choi, J.-Y. Kim, S.B. Yoon, K. Song and Y.J. Kim, Microchem. J., 99, 332 (2011).

5. J.-Y. Kim, Y.S. Choi, Y.J. Park, K. Song, S.-H. Jung and E.M.A. Hussein, Appl. Radiat. Isot., 69, 1241 (2011).

6. R.V. Siriwardane, M.S. Shen, E.P. Fisher and J.A. Poston, Energy Fuels, 15, 279 (2001).

7. L. Monser and N. Adhoum, Sep. Purif. Technol., 26, 137 (2002).

8. S. Bashkova, F.S. Baker, X. Wu, T.R. Armstrong and V. Schwartz, Carbon, 45, 1354 (2007).

9. D.O. Cooney, A. Nagerl and A.L. Hines, Water Res., 17, 403 (1983).

10. J.E. Fernandez, V. Scot, E.D. Giulio and L. Verardi, X-Ray Spectrom., 40, 101 (2011).

11. S.A. Margolis and P.H. Huang, X-ray Absorption and Diffraction, Encyclopedia of Analytical Science, Elsevier Ltd., pp. 365-377 (2005). 